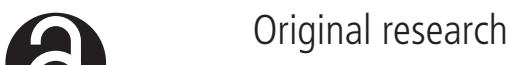

\section{Mass eradication of Helicobacter pylori to reduce gastric cancer incidence and mortality: a long-term cohort study on Matsu Islands}

\author{
Tsung-Hsien Chiang, ${ }^{1,2,3}$ Wei-Jung Chang, ${ }^{4}$ Sam Li-Sheng Chen, ${ }^{5}$ \\ Amy Ming-Fang Yen (D) ,' Jean Ching-Yuan Fann, ${ }^{6}$ Sherry Yueh-Hsia Chiu (1) , 7,8 \\ Yi-Ru Chen, ${ }^{9}$ Shu-Ling Chuang, ${ }^{4,10}$ Chun-Fu Shieh, ${ }^{11}$ Cheng-Ying Liu, ${ }^{12}$ \\ Han-Mo Chiu (10 , 1,4 Hung Chiang, ${ }^{13}$ Chia-Tung Shun, ${ }^{14,15}$ Ming-Wei Lin, ${ }^{16}$ \\ Ming-Shiang Wu (D) , Jaw-Town Lin (D) , ${ }^{1,17}$ Chang-Chuan Chan, ${ }^{18,19}$ \\ David Y Graham (D) , ${ }^{20}$ Hsiu-Hsi Chen (D) , ${ }^{4,19}$ Yi-Chia Lee (D) 1,4,10,19
}

- Additional material is published online only. To view please visit the journal online (http://dx.doi.org/10.1136/ gutjnl-2020-322200).

For numbered affiliations see end of article.

Correspondence to Dr Yi-Chia Lee, Department of Internal Medicine, College of Medicine, National Taiwan University, Taipei, Taiwan: yichialee@ntu.edu.tw

$\mathrm{H}-\mathrm{HC}$ and $\mathrm{Y}-\mathrm{CL}$ contributed equally.

Received 12 June 2020 Revised 11 July 2020 Accepted 12 July 2020 Published Online First 13 August 2020
Check for updates

(C) Author(s) (or their employer(s)) 2021. Re-use permitted under CC BY-NC. No commercial re-use. See rights and permissions. Published by BMJ.

To cite: Chiang T-H, Chang W-J, Chen SL-S, et al. Gut 2021;70:243-250.

\section{ABSTRACT \\ Objective Although mass eradication of Helicobacter} pylori has been proposed as a means to eliminate gastric cancer, its long-term effects remain unclear.

Design Mass eradication of $H$. pylori infection was launched in 2004 and continued until 2018 for a high-risk Taiwanese population aged 30 years or older dwelling on Matsu Islands with prevalent $H$. pylori infection. Test positives for the ${ }^{13} \mathrm{C}$-urea breath test underwent eradication therapy. We evaluated the effectiveness of the mass eradication in reducing two main outcomes, incidence and mortality rates of gastric cancer, until the end of 2016 and 2018, respectively.

Results After six rounds of mass screening and eradication, the coverage rate reached $85.5 \%$ (6512/7616). The referral rate for treatment was $93.5 \%$ (4286/4584). The prevalence rates of $H$. pylori fell from $64.2 \%$ to $15.0 \%$ with reinfection rates of less than $1 \%$ per person-year. The presence and severity of atrophic gastritis and intestinal metaplasia also decreased with time. Compared with the historical control period from 1995 to 2003, the effectiveness in reducing gastric cancer incidence and mortality during the chemoprevention period was 53\% $(95 \% \mathrm{Cl} 30 \%$ to $69 \%, p<0.001)$ and $25 \%(95 \% \mathrm{Cl}-14 \%$ to $51 \%$, $p=0.18)$, respectively. No significant changes were noted in the incidence rates of other digestive tract cancers or the antibiotic resistance rate of $H$. pylori.

Conclusion Population-based eradication of $H$. pylori has significantly reduced gastric cancer incidence with no increase in the likelihood of adverse consequences. A significant reduction in mortality is likely to be achieved with a longer follow-up period.

Trial registration number NCT00155389

\section{INTRODUCTION}

Gastric cancer is the third-leading cause of cancer death worldwide, accounting for $>800000$ deaths every year. ${ }^{12}$ Most cases are a consequence of infection with Helicobacter pylori and are of one of the cancers associated with inflammation. Typically, the cancer develops after a long period of a multistep

\section{Significance of this study}

What is already known on this subject?

- Randomised controlled trials based on hospital patients have suggested that the risk of gastric cancer can be reduced by about $50 \%$ in Helicobacter pylori eradicated patients as compared with non-eradicated patients.

- Experts have reached consensus that mass screening and eradication of $H$. pylori should be adopted as a public health policy to eliminate the threat from gastric cancer for high-risk populations.

- However, there is a lack of direct evidence to confirm the long-term benefits of a mass eradication programme and it remains unclear whether the intervention will also lead to an increased risk of adverse consequences.

process from chronic gastric inflammation, mucosal atrophy, metaplastic epithelia, intraepithelial neoplasia and finally to invasive adenocarcinoma. ${ }^{3}$ Eradication of $H$. pylori results in healing of inflammation and halts progression of the $H$. pylori-associated mucosal and genetic damages. ${ }^{4}$ The effect of timing of $H$. pylori eradication on cancer risk is best visualised by the inspection of the age-related increase in gastric cancer risk, which shows that initially there is a long latent period of low risk followed by a period with an exponentially increased risk. The overall effectiveness of $H$. pylori eradication in terms of cancer prevention is thus thought to depend on when in the chain of progression the infection is eradicated.

The possibility of eliminating or greatly reducing the threat of gastric cancer by eradication of $H$. pylori has increasingly gained attention. ${ }^{5}$ The fact that clinical trials based on hospital patients confirmed the efficacy of antibiotic treatment in gastric cancer prevention ${ }^{6-9}$ has led to recommendations for mass screening and $H$. pylori eradication of high-risk populations. ${ }^{1011}$ However, adoption of this strategy 
Significance of this study

What are the new findings?

- Mass H. pylori eradication programme has been implemented in a high gastric cancer risk community since 2004, resulting in a significant reduction in the occurrence of gastric cancer by $53 \%$ while taking into consideration the participation rate for screening, the referral-to-treatment rate, an increase in newly enrolled eligible subjects, and the natural improvement, other than $H$. pylori eradication, in the gastric cancer incidence.

- There was lacking of significant change in the incidence rate of oesophageal cancer or colorectal cancer. The antibiotic resistance rate of $H$. pylori was unchanged through the selection of effective eradication regimens and retesting those who had completed the treatments.

- Following the declining trend data, the predicted gastric cancer incidence rate will be $<6$ per 100000 person-years in 2023 and a significant 39\% reduction in gastric cancer mortality will be achieved by 2025 .

How might it impact on clinical practice in the foreseeable future?

- Mass screening and eradication of $H$. pylori can effectively reduce the gastric cancer incidence, which would soon make this cancer a rare event, significantly reduce the number of related deaths, and reach the final goal of eliminating the threat from gastric cancer.

as a healthcare policy has remained controversial because the long-term effects, including both benefits and potential drawbacks, remain unclear. For example, prior to the actual treatment, any mass eradication programme must include multiple steps (invitation, participation, testing and referral), which may lower the magnitude of observed benefit from that expected based on clinical trial data. ${ }^{12}$ In addition, any mass eradication service programme needs to recognise that the population is a dynamic cohort, rather than a fixed group of patients, requiring continuous entrance of screening-naïve individuals, who may carry a higher risk of $H$. pylori, increase the risk of reinfection in the post-treatment subjects and reduce the treatment effectiveness. ${ }^{13}$ In addition to benefits, there are a number of theoretical harms. For example, because eradication of $H$. pylori infection can restore or improve gastric acid secretion, it has been postulated that it may increase acid reflux to the oesophagus and even lead to an increase in oesophageal adenocarcinoma. ${ }^{14}$ Antibiotic treatment also has the potential to disrupt the gut microbiota, which might produce unknown consequences. ${ }^{15}$ Finally, there is concern regarding its effect on global antimicrobial resistance or emergence of antibiotic-resistant strains of H. pylori. ${ }^{16}{ }^{17}$ These potentially collateral effects of $H$. pylori eradication are largely theoretical and real-world evidence is urgently needed to throw light on the benefits and harms.

A mass $H$. pylori eradication programme was launched in a high-risk area for gastric cancer in 2004 in Taiwan. ${ }^{18}$ Short-term benefits of reducing the risk of $H$. pylori infection and premalignant gastric lesions were evident around 5 years after the intervention and a significant reduction in gastric cancer incidence was anticipated after long-term follow-up. ${ }^{19}$ The programme has been continued with the main objective of evaluating the long-term benefits of mass eradication in reducing the gastric cancer incidence and gastric cancer-specific mortality. This would be obtained by comparing data from the pre-eradication and posteradication periods (1995-2003 and 2004-2016/2018, respectively) in the same population while adjusting for the decline in gastric cancer incidence due to improvements in sanitation and hygiene (called the 'historical effect'). In addition to quantifying the benefits, we also assessed what sort, if any, of the speculated adverse effects occurred.

\section{METHODS}

\section{Study population and preventive programme}

The eligible population consisted of the inhabitants of the Matsu Islands in Taiwan aged $\geq 30$ years and registered in the Population Registry. Owing to the high risk of gastric cancer in this population, an initial screening programme was implemented in 1995-1998 based on pepsinogen measurements. ${ }^{20}$ Mass screening and eradication of $H$. pylori were launched in 2004, the short-term benefits were evaluated in 2008, and the programme has been fully implemented on a biennial basis since 2012. Repeated screening was done to deal with the dynamic nature of this cohort and the observed increase in eligible subjects.

The timeline for the screening process and programme implementation are shown in figure 1. In brief, during May to June, the mass screening programme was initiated by the first-line staff in Health Bureau of Lienchiang County on Matsu Islands. Eligible inhabitants were invited by mail, telephone or announcement in social media and newspapers to receive screening for $\mathrm{H}$. pylori with the ${ }^{13} \mathrm{C}$-urea breath test. Pregnant or lactating women, patients with major comorbid diseases, and those who had undergone gastric surgery were excluded. Participants' demographic data, social habits and medical history were recorded in a structured questionnaire. Those testing positive received eradication treatment, which was designed by the core members of the study team (T-HC and Y-CL) and prescribed by the physicians in Lienchiang County Hospital on Matsu Islands. Initially, the prescribed regimen was $7-14$ days triple therapy consisting of $40 \mathrm{mg}$ of esomeprazole once a day, $1 \mathrm{~g}$ of amoxicillin two times a day, and $500 \mathrm{mg}$ of clarithromycin two times a day. The updated regimen since 2012 was a 10-day sequential therapy consisting of $30 \mathrm{mg}$ of lansoprazole and $1 \mathrm{~g}$ of amoxicillin twice a day for days $1-5$, followed by $30 \mathrm{mg}$ of lansoprazole, $500 \mathrm{mg}$ of clarithromycin and $500 \mathrm{mg}$ of metronidazole two times a day for days $6-10$. Those who failed initial treatment were retreated with a 10-day triple therapy consisting of $40 \mathrm{mg}$ of esomeprazole once a day, $1 \mathrm{~g}$ of amoxicillin two times a day and $500 \mathrm{mg}$ of levofloxacin once a day. The eradication rates were $85.3 \%$ and $91.4 \%$ for the first-line and second-line treatments, respectively. ${ }^{18} 21$

\section{Study design for evaluation}

To evaluate the long-term benefits and to take into consideration the improved sanitation and hygiene during the long study period, which may result in the improvement of the gastric cancer incidence other than the effect from $H$. pylori eradication, we applied a 'before and after' study design. ${ }^{22}$ The data on the incidence and mortality rates of gastric cancer between 1995 and the end of 2003 (historical control data) were used for the following analysis with a Poisson model to predict the occurrence of incident gastric cancer and that of gastric cancer-specific death after 2004, respectively, in the absence of screening. We then compared the expected number of cases with the number observed during the chemoprevention period to determine the magnitude of effectiveness. 
Matsu population aged $\geq \mathbf{3 0}$ years

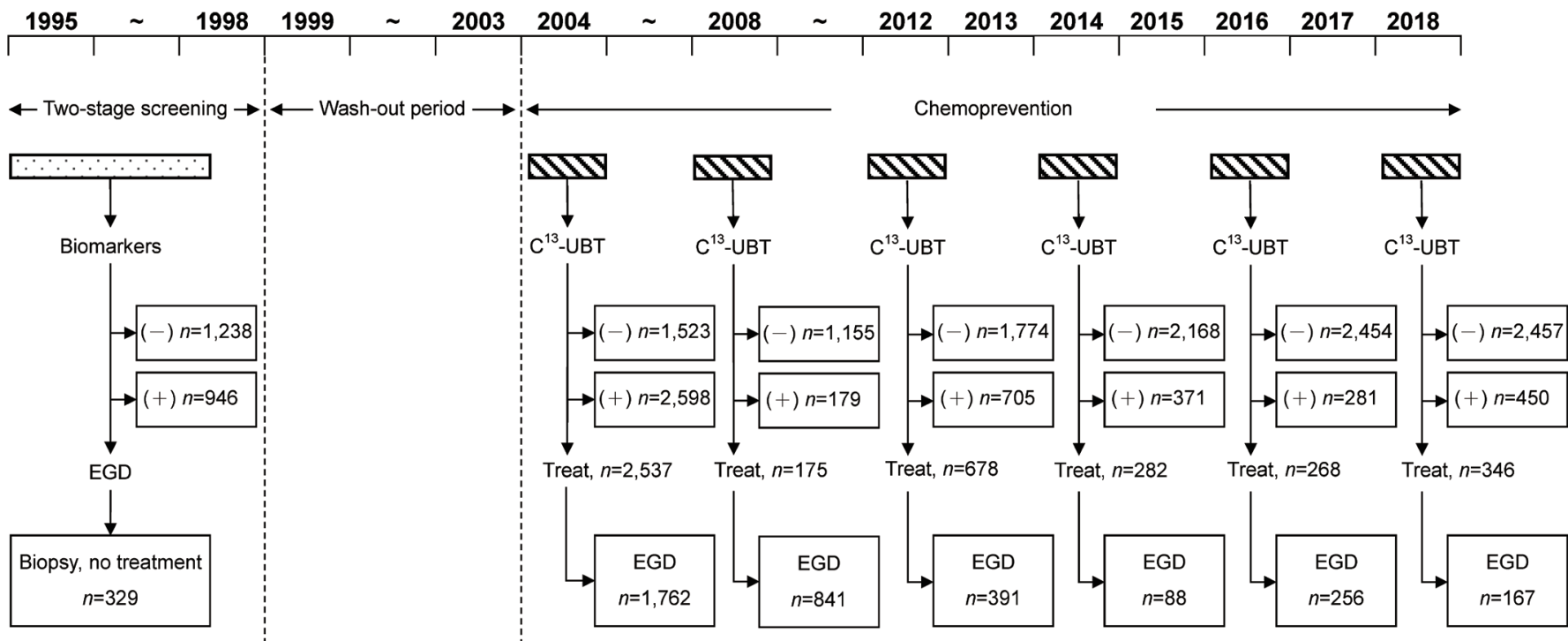

Pepsinogen test, H. pylori antibody, and endoscopic examination (1995 $\rightarrow$ 1998)

\$ Test and treat for $H$. pylori infection, and endoscopic examination $(2004,2008,2012,2014,2016$, and 2018)

Figure 1 Timeline of the gastric cancer prevention programme implemented on the Matsu Islands, Taiwan. EGD, oesophagogastroduodenoscopy; UBT, urea breath test.

\section{Endoscopic examination}

In addition to identifying any lesions suspicious of being cancerous, endoscopic examination was used to evaluate the prevalence and severity of the premalignant gastric lesions. Their changes over time were used to support our main findings of gastric cancer risk reduction. During September to October, about 4 months after the mass eradication programme, endoscopic examination was performed by a team of experienced endoscopists from National Taiwan University Hospital (Taipei, Taiwan). Biopsy specimens were routinely taken from gastric mucosae in the antrum (from the greater and lesser curvatures $2-3 \mathrm{~cm}$ from the pylorus) and corpus (one each from the lesser and greater curvature at the middle corpus), using a modified Sydney protocol. ${ }^{23}$ Sampling was done at the same locations in all subjects to maintain consistency. A senior histopathologist (T-HC), blinded to participants' clinical status and test results, performed all histological assessments. Specimens were graded, using the Updated Sydney Classification, as acute inflammation (polymorphonuclear infiltrates), chronic inflammation (lymphoplasmacytic infiltrates), atrophic gastritis (loss of glandular tissue and fibrous replacement) or intestinal metaplasia (presence of goblet cells and absorptive cells). The severity of each category was rated as none, mild, moderate or marked, so as to classify the severity of premalignant lesions using Operative Link for Gastritis Assessment of Atrophic Gastritis (OLGA) and Operative Link for Gastritis Assessment of Intestinal Metaplasia (OLGIM) criteria. $^{2425}$

Also, we performed an antibiotic susceptibility testing for $H$. pylori isolates to determine the presence of antibiotic resistance. Biopsy specimens were cultured on plates containing Brucella chocolate agar with 7\% sheep blood and incubated for 14 days under microaerobic conditions. The minimum inhibitory concentrations were determined by agar dilution test in the laboratory of National Taiwan University Hospital. The resistance cut-off values for amoxicillin, clarithromycin, levofloxacin, metronidazole and tetracycline were defined as greater than 0.5 , $1,1,8$ and $0.5 \mathrm{mg} / \mathrm{L}$, respectively. Surveys of antibiotic resistance studies were carried out in 2014 and $2018 .^{21}$

\section{Outcomes}

The primary outcome was the incidence rate of gastric cancer. The secondary outcome was the mortality rate from gastric cancer. Outcomes were ascertained according to the National Cancer and Mortality Registries until the end of 2016 and 2018, respectively, because there was a time lag of 2 years when these two statistics were available. ${ }^{2627}$ These annual reports were characterised by the high coverage (>99\%; hospitals in Taiwan were instructed to report all cases of cancers) and high accuracy (ie, the percentage of death-certificate-only cases was $<1 \%) .{ }^{28}$ The number of those at risk was determined by searching the databases of the Household Registration Administration System. ${ }^{29}$ Since all these measures were based on the entire population in Taiwan, the rate of lost to follow-up of our cohort was negligible. Histopathology was classified according to the criteria of WHO. ${ }^{30}$

\section{Statistical analysis}

For the baseline characteristics of participants, categorical data were expressed as a percentage $(\%)$ and continuous data was expressed as mean (SD). We first determined the prevalence rate and reinfection rate of $H$. pylori; the former was calculated as the number of positive test results divided by the number of participants and the latter as the number of positive test results divided by the person-years of follow-up for those who had received successful eradication treatment. Second, we calculated the presence and severity of premalignant gastric lesions using the OLGA and OLGIM systems and evaluated the changes over time using the Cochran-Armitage trend test. Third, to quantify the benefit of reducing the incidence of gastric cancer, we applied a 


\begin{tabular}{|c|c|c|c|c|c|c|}
\hline Baseline variables & 2004 & 2008 & 2012 & 2014 & 2016 & 2018 \\
\hline No of participants & 4121 & 1338 & 2479 & 2539 & 2735 & 2907 \\
\hline No of Helicobacter pylori infection (\%) & $2598(63.0)$ & $179(13.4)$ & 705 (28.4) & $371(14.6)$ & $281(10.3)$ & $450(15.5)$ \\
\hline Mean age, year (SD) & $59.1(13.6)$ & $52.8(11.0)$ & $50.9(12.7)$ & $51.9(12.9)$ & $52.3(13.2)$ & $52.7(13.2)$ \\
\hline Male sex, $\%$ & 48.7 & 49.7 & 48.3 & 48.7 & 49.4 & 49.2 \\
\hline No of new participant (\%) & $4121(100)$ & $89(6.7)$ & $945(38.1)$ & $494(19.5)$ & $402(14.7)$ & $461(15.9)$ \\
\hline No of $H$. pylori infection in new participants (\%) & $2598(63.0)$ & $41(46.1)$ & $474(50.2)$ & $195(39.5)$ & $146(36.3)$ & $182(39.5)$ \\
\hline No of endoscopy & 1762 & 841 & 391 & 88 & 256 & 167 \\
\hline Active peptic ulcer, $\%$ & $193(11.0)$ & $30(3.6)$ & $68(17.3)$ & $14(15.9)$ & $34(7.5)$ & $5(3.0)$ \\
\hline Gastric cancer (\%) & $9(0.5)$ & $1(0.1)$ & $3(0.8)$ & $2(2.3)$ & $2(0.8)$ & $2(1.2)$ \\
\hline Reflux oesophagitis (\%) & $241(13.7)$ & $230(27.3)$ & $97(24.8)$ & $26(29.5)$ & $61(23.8)$ & $36(21.6)$ \\
\hline Barrett's oesophagus (\%) & 0 & $2(0.2)$ & $3(0.8)$ & 0 & $1(0.4)$ & $1(0.6)$ \\
\hline Oesophageal cancer (\%) & 0 & 0 & 0 & $1(1.1)$ & 0 & 0 \\
\hline
\end{tabular}

Bayesian Gamma-Poisson conjugated model to first analyse the time trend of the prechemoprevention period (1995-2003) that was used to build up the historical control group, making allowance for the declining trend due to self-improvement as indicated above. ${ }^{31}$ We updated the parameters of posterior Gamma distribution annually until 2003. These parameters estimated from prechemoprevention period were further used to estimate the expected number of cases of gastric cancer in comparison with the observed cases given the underlying person-years after chemoprevention (2004-2016). The effectiveness of H. pylori treatment in reducing the gastric cancer incidence was calculated as: (1-observed/expected number $) \times 100 \%$, with the corresponding 95\% CI. Regarding the effectiveness of reducing gastric cancer-specific death, the calculation was carried out in the same manner until the end of 2018.

We also used the Poisson change point (the interrupted time with chemoprevention and endoscopy) regression model ${ }^{31}$ to extrapolate the above trends to 2025 and to make a prediction of when the gastric cancer incidence would be $<6$ per 100000 person-years, which is defined as the goal of eliminating the threat from gastric cancer (or making gastric cancer become a rare disease). ${ }^{32}$

Finally, we explored the risks of adverse consequences using two approaches. First, we evaluated the trends in oesophageal and colorectal cancer incidence rates in the same population, using the reference year of 2004, when chemoprevention started. Second, we made a comparison with the $H$. pylori antibiotic resistance rate between 2014 and 2018, when the programme was fully implemented.

Basic statistical analyses were performed by using SAS V.9.4 (SAS Institute). The computer software of WinBUGs was used for Bayesian analyses. All $\mathrm{p}$ values were two sided, and $\mathrm{p}$ values $<0.05$ were considered statistically significant.

\section{Patient and public involvement}

Members of the public (C-FS and C-YL), who developed the policy and provided administrative support, were involved in the present study to be coauthors.

\section{RESULTS}

\section{Attendance and referral rates}

The total number of residents based on the population-based registry grew from 9359 in 2004 to 12536 in 2016 (online supplementary table 1). After the initial rounds in 2004 $(n=4121)$ and $2008 \quad(n=1334$, mainly repeated screenees), there were $2479,2539,2735$, and 2907 subjects who had the uptake of screen in 2012, 2014, 2016 and 2018, respectively (figure 1). The overall coverage rate given the target population aged 30 years or older was 85.5\% (6512/7616). During the full implementation period, the demographic data were similar, with an average age of around 50 years and 50\% for both sexes (table 1). For subjects testing positive for H. pylori, the overall referral rate for the antibiotic treatment was $93.5 \%$ (4286/4584).

Among subjects who received upper endoscopic examinations, the prevalence rates of active peptic ulcer declined from $17.3 \%$ in 2012 to $3.0 \%$ in 2018 . The results for reflux oesophagitis, after an initial increase in 2008 mainly based on the repeated screenees, were similar around 25\% during the full implementation period. Histologically confirmed Barrett's oesophagus was consistently a rare diagnosis. The number of those with a screening-detected gastric cancer $(n=19)$ was much higher than that of oesophageal cancer $(n=1)$.

\section{Changes in $H$. pylori infection and the reinfection rates}

As shown in figure 2, the prevalence rates of $H$. pylori infection declined from $64.2 \%$ in 2004 to $15.0 \%$ in 2008 during the initial period. Again, the prevalence rates declined from $28.3 \%$ to $15.7 \%$ during the full implementation period (2012-2018). The reinfection rate was consistently low, with the initial result of 0.95 per 100 person-years in 2008 and follow-up results of $0.34,0.36,0.29$ and 0.74 per 100 person-years evaluated in 2012, 2014, 2016 and 2018, respectively.

It should be noted that new participants continuously expanded the number of eligible subjects. As shown in table 1 , there were 89 (6.7\%), 945 (38.1\%), 494 (19.5\%), 402 (14.7\%) and 461 (15.9\%) new participants in 2008, 2012, 2014, 2016 and 2018, respectively; among these, the infection rates of $H$. pylori were high at 46.1\%, 50.2\%, 39.5\%, 36.3\% and 39.5\%, respectively.

\section{Changes in prevalence rates of premalignant gastric lesions}

As shown in figure 3, for both the presence of atrophic gastritis (ie, OLGA grade $\geq 1$ ) and advanced-stage atrophic gastritis (ie, OLGA grade 3 or 4 ), there was a significant decline in prevalence rates (both $\mathrm{p}<0.001$ ). Significant decreases were similarly seen in the prevalence rates of both intestinal metaplasia (ie, OLGIM grade $\geq 1$ ) and advanced-stage intestinal metaplasia (ie, OLGIM grade 3 or 4 ), although the magnitude was lower than that of atrophic gastritis. 


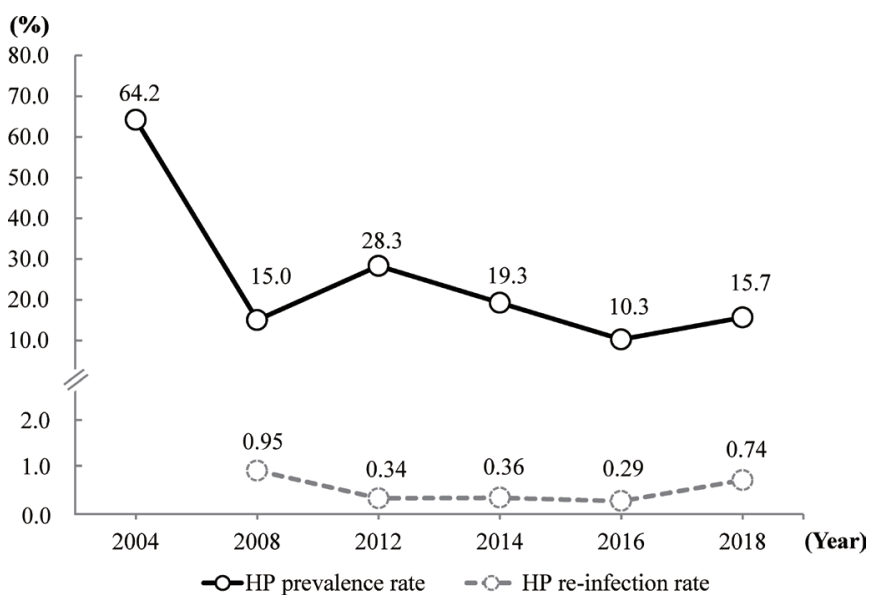

Figure 2 Prevalence rates and reinfection rates of Helicobacter pylori (HP).

\section{Changes in incidence and mortality rates of gastric cancer}

Evaluation of short-term benefits in 2008 showed that both $H$. pylori infection and atrophic gastritis were significantly reduced by $79 \%$ and $61 \%$, respectively. As reported previously, the reduction in gastric cancer was $25 \%(\mathrm{p}=0.21)$ despite a short follow-up period. ${ }^{19}$

Regarding the long-term benefits, as shown in figure 4, the gastric cancer incidence continuously declined with time. Estimation based on the historical control from 1995 to 2003, as opposed to the observed number during the chemoprevention period from 2004 to 2016 (online supplementary table 1), yielded an effectiveness in reducing gastric cancer incidence of $53 \%$ (95\% CI $30 \%$ to $69 \%, \mathrm{p}<0.001)$. When we extrapolated the decreasing trend, an incidence rate of $<6$ per 100000 person-years would occur in 2023 and a greater reduction of
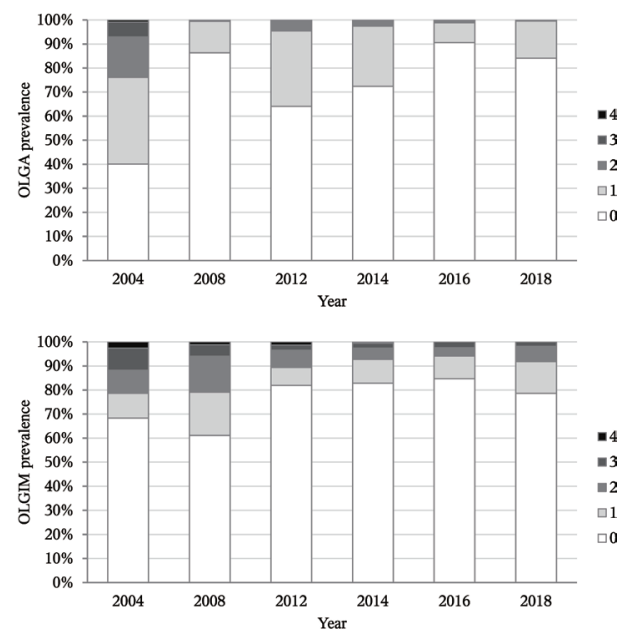

Figure 3 The presence and severity of premalignant gastric lesions according to the operative link for gastritis assessment of atrophic gastritis (OLGA) and operative link for gastritis assessment of intestinal metaplasia (OLGIM) grading systems. over 2004-2018, for the presence of atrophic gastritis and intestinal metaplasia (ie, grade $\geq 1$ ), the prevalence rates declined from $55.9 \%$ to $15.9 \%$ and from $31.7 \%$ to $21.4 \%$, respectively (both $p<0.001$ ). For advanced-stage atrophic gastritis and intestinal metaplasia (ie, grade 3 or 4), the prevalence rates declined from $7.1 \%$ to 0 and from $11.8 \%$ to $1.8 \%$, respectively (both $\mathrm{p}<0.001$ ).

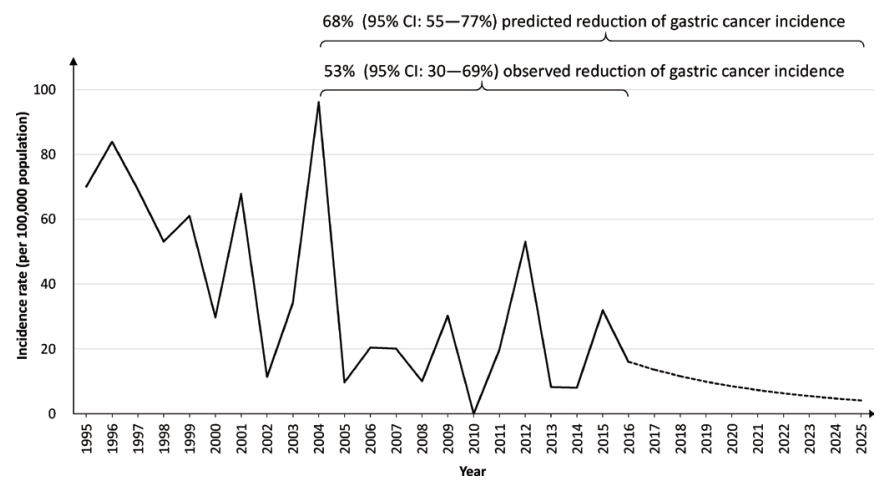

Figure 4 Incidence rates of gastric cancer between 1995 and 2016, correlated with the start of the mass eradication programme in 2004. The dash line indicates the predicted trend to 2025. The GammaPoisson regression model was internally validated by using data on the incidence rate of gastric cancer between 1995 and 2003 with the goodness-of-fit test $\left(\chi^{2}=4.95 ; p=0.84\right)$. The magnitude of risk reduction was determined by comparing the expected number of cases with the number observed during the chemoprevention period.

$68 \%$ (95\% CI 55\% to 77\%, p<0.001) would be expected by 2025.

The results of gastric cancer mortality rates are shown in figure 5. Estimation based on the historical control from 1995 to 2003, as compared with the observed number during the period of chemoprevention from 2004 to 2018 (online supplementary table 2), yielded a non-significant 25\% (95\% CI - 14\% to 51\%, $\mathrm{p}=0.18$ ) reduction of the gastric cancer mortality rate. When we extrapolated the decreasing trend, a significant reduction of $39 \%$ (95\% CI $12 \%$ to $57 \%, \mathrm{p}=0.007$ ) would be achieved by 2025 .

\section{Changes in incidence rates of other digestive tract cancers} Incidence rates of oesophageal cancer and colorectal cancer are shown in online supplementary figure 1. Comparison of the incidence rates before and after the mass eradication programme revealed that there were non-significant changes of $22 \%(95 \%$ CI $-39 \%$ to $83 \%, p=0.60)$ and $23 \%(95 \%$ CI $-10 \%$ to

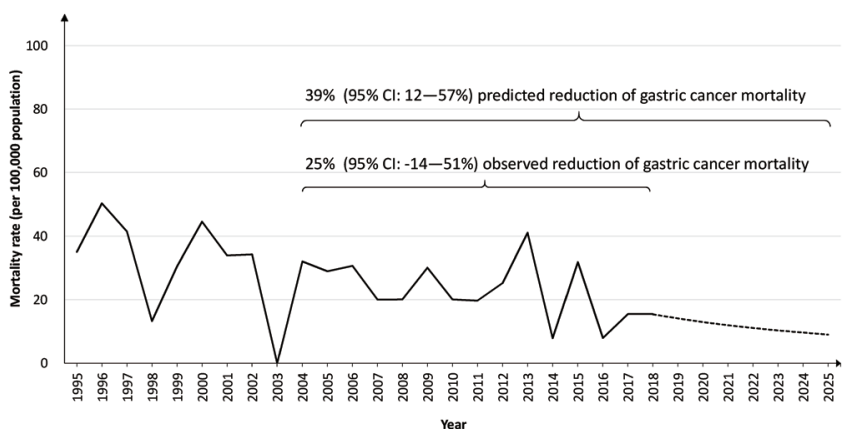

Figure 5 Rates of mortality from gastric cancer between 1995 and 2018 , correlated with the start of the mass eradication programme in 2004. The dash line indicates the predicted trend to 2025. The GammaPoisson regression model was internally validated by using data on the mortality rate of gastric cancer between 1995 and 2003 with the goodness-of-fit test $\left(\chi^{2}=3.88 ; p=0.92\right)$. The magnitude of mortality reduction was determined by comparing the expected number of cases with the number observed during the chemoprevention period. 
$56 \%, \mathrm{p}=0.30)$ for oesophageal cancer and colorectal cancer, respectively.

\section{Changes in antibiotic resistance rates of $H$. pylori}

In 2014, the H. pylori resistance rates to amoxicillin, clarithromycin, metronidazole, levofloxacin and tetracycline were $0.8 \%$ (3/369), 9.2\% (34/368), 21.3\% (78/367), 8.4\% (31/369) and $4.1 \%(15 / 367)$, respectively; the results in 2018 were $1.0 \%$ (2/196), 10.2\% (20/196), 22.4\% (44/196), 10.2\% (20/196) and $4.1 \%(8 / 196)$, respectively. The antibiotic resistance rates of $H$. pylori for the four successive screening rounds during full implementation did not have a significant change $(\mathrm{p}=0.46)$.

\section{DISCUSSION}

The present study quantified the benefits of mass screening and eradication of $H$. pylori infection in an area with highly endemic $H$. pylori infection and a high incidence rate of gastric cancer. In an organised programme setting with a quality-assured screening, a significant reduction in $H$. pylori infection was accompanied by declines in premalignant gastric lesions and gastric cancer. This remarkable effectiveness persisted after adjustment for the declining trend of gastric cancer incidence due to improvements in sanitation and hygiene. The project suggests that the results will further lead to a significant reduction in mortality from gastric cancer and ultimately achieve the goal of eliminating the gastric cancer burden in this area.

Traditional gastric cancer prevention is based on endoscopic screening to identify early-stage gastric cancer so that treatment will still be effective; for example, universal endoscopic screening reduced the risk of dying from gastric cancer by $21 \%$ in Republic of Korea. ${ }^{33}$ Compared with endoscopic screening, mass screening and eradication of $H$. pylori are designed to modify the natural course of the disease, (ie, the goal of this strategy is to prevent the future risk of gastric cancer). Our finding of a $53 \%$ risk reduction is similar to the results from other primary prevention trials in patients who received endoscopic resection of early-stage gastric cancer $(50 \%),{ }^{67}$ in patients with premalignant lesions $(52 \%)^{8}$ and in first-degree relatives of patients with gastric cancer $(55 \%))^{9}$ The result is further supported by the results from several meta-analyses that include either randomised trials or cohort studies (about 50\% reduction in incidence rates). ${ }^{3435}$ Second, by reducing dependence on the limited supply of endoscopists, mass screening and eradication can be initiated by the first-line staff in public health units, clinics or hospitals, making this programme easier and cheaper to apply than late-stage interventions. Third, $H$. pylori eradication therapy can provide additional benefits by treating or preventing $\mathrm{H}$. pylori-induced dyspepsia or peptic ulcers, making this approach more cost-effective on population level. ${ }^{36}$

Although mass eradication would be of the greatest benefit in regions with high gastric cancer risk, ${ }^{34}$ it is important to note that intermediate-risk or low-risk countries also contain subpopulations at higher risk who are candidates for screening. Such a scenario has been demonstrated in Matsu Islands, where the incidence rate of gastric cancer is much higher than that on the main island of Taiwan and similar to that of high-risk coastal provinces of China such as Fujian and Shandong. ${ }^{8738}$ In addition, we found that, even when the programme had eliminated the infection from the original inhabitants, immigrants with the H. pylori infection were still potentially able to reintroduce the pathogen into the posteradication population. Therefore, as a healthcare policy, active screening of newcomers and monitoring postscreen subjects for reinfection may be required to maintain the reinfection rate below the threshold value (eg, 2.2 per 100 person-years), in order to maintain the relative cost-effectiveness of the programme as compared with the endoscopic approach. ${ }^{13}$

One prediction by the International Agency for Research on Cancer indicated that, based on the historical data, the declining trend in gastric cancer incidence tends to be very slow, especially in high-risk or intermediate-risk countries. ${ }^{32}$ For example, in Japan, Chile, Lithuania and China, the age-standardised incidence rates of gastric cancer, taking into consideration population growth and ageing, are estimated to decline from 36.3, $21.3,14.8$ and 13.1 to $30.4,20.8,12.6$ and 10.3 per 100000 person-years, respectively, until the end of 2035. Moreover, in Republic of Korea, the incidence rate is expected to increase from 40.8 to 43.4 per 100000 person-years from 2010 to 2035 . These results indicate that, without active intervention, gastric cancer will not become a rare disease in countries with higher disease burden in the foreseeable future. ${ }^{39}$ Our study suggests that this goal is achievable more rapidly through mass screening and eradication.

Hypotheses have been raised regarding possible adverse consequence of $H$. pylori eradication. For example, animal studies have suggested that antibiotic exposure may disrupt the earlylife gut microbiome, cause a loss of biodiversity, and potentially lead to metabolic perturbations in adiposity, bone growth or immunological development. ${ }^{40}$ However, causative associations are lacking, and the proposed programme is designed for adults. Long-term follow-up of our cohort failed to reveal an increase in the incidence of other digestive tract cancers, presumably the sites most vulnerable to the dysbiosis associated with antibiotic treatment.

The strengths of our study include the organised delivery of the screening service, the rigorous efforts made to maintain the programme on a regular basis, and the ascertainment of outcomes through an accurate registration system. Even though the number of participants in our study population was relatively small, targeting high-risk subpopulations is more applicable and generalisable as a healthcare policy than a wide treatment of the population as a whole. ${ }^{41}$ However, our study has limitations. The major limitation is that it was not a randomised controlled trial that generated groups with comparable baseline profiles to validate the effects of the eradication treatment; ethical considerations in this high-risk area precluded the use of such a study design. Nonetheless, using the long-term historical data, we simulated a control arm close to the trend without intervention. Our risk reduction of $53 \%$ based on the comparison between observed and expected data was more conservative than the direct comparison of incidence rates between the prechemoprevention and postchemoprevention periods (56.9 vs 19.1 per 100000 person-years, a risk reduction of 66\%), because it took into account the self-improvement in gastric cancer incidence. We, therefore, believe that our findings likely approximate the results of a randomised controlled trial. Second, we found that the risk of short-course antibiotics to produce resistant strains of H. pylori was minimal, likely because we selected effective eradication therapies and retested those who had completed either the first-line or the second-line treatment. ${ }^{212}$ However, our abilities to translate this finding to other human bacteria are limited, although recent studies have indicated that within participating individuals, such changes are transient and may even provide beneficial effects to the gut microbiota. ${ }^{434}$ Finally, although we demonstrated that gastric cancer can become as a rare disease through active intervention, $H$. pylori eradication does not reset the population risk to zero but rather stops and possibly lowers the individual-specific increase in risk. Those who remain at 
higher risk (eg, advanced-stage atrophic gastritis and intestinal metaplasia) may benefit from surveillance to accurately define the personal risk and allocate endoscopic resources. ${ }^{45} 46$

In conclusion, we demonstrated that mass screening and eradication of $H$. pylori infection is associated with a significant reduction in gastric cancer incidence, without evidence of an increase in adverse consequences. The results support the population-wide application of this approach, particularly in areas with a high prevalence rate of $H$. pylori infection and a high incidence rate of gastric cancer, to eliminate the threat from this deadly cancer.

\section{Author affiliations}

${ }^{1}$ Department of Internal Medicine, College of Medicine, National Taiwan University, Taipei, Taiwan

${ }^{2}$ Department of Integrated Diagnostics and Therapeutics, National Taiwan University Hospital, Taipei, Taiwan

${ }^{3}$ Graduate Institute of Clinical Medicine, College of Medicine, National Taiwan University, Taipei, Taiwan

${ }^{4}$ Graduate Institute of Epidemiology and Preventive Medicine, College of Public Health, National Taiwan University, Taipei, Taiwan

${ }^{5}$ School of Oral Hygiene, College of Oral Medicine, Taipei Medical University, Taipei, Taiwan

${ }^{6}$ Department of Health Industry Management, Kainan University, Taoyuan, Taiwan ${ }^{7}$ Department of Health Care Management and Healthy Aging Research Center, Chang Gung University, Taoyuan, Taiwan

${ }^{8}$ Division of Hepatogastroenterology, Department of Internal Medicine, Kaohsiung Chang Gung Memorial Hospital, Kaohsiung, Taiwan

${ }^{9}$ Department of Health Care Management, National Taipei University of Nursing and Health Sciences, Taipei, Taiwan

${ }^{10}$ Department of Medical Research, National Taiwan University Hospital, Taipei, Taiwan

${ }^{11}$ Health Bureau of Lienchiang County, Nangan Township, Lienchiang County, Matsu, Taiwan

${ }^{12}$ Lienchiang County Government, Nangan Township, Lienchiang County, Matsu, Taiwan

${ }^{13}$ Taipei Institute of Pathology, Taipei, Taiwan

${ }^{14}$ Department of Pathology, National Taiwan University Hospital, Taipei, Taiwan

${ }^{15}$ Department and Graduate Institute of Forensic Medicine, College of Medicine, National Taiwan University, Taipei, Taiwan

${ }^{16}$ Institute of Public Health, National Yang-Ming University, Taipei, Taiwan

${ }^{17}$ Center for Digestive Medicine, China Medical University Hospital, Taichung, Taiwan ${ }^{18}$ Institute of Environmental and Occupational Health Sciences, College of Public Health, National Taiwan University, Taipei, Taiwan

${ }^{19}$ Innovation and Policy Center for Population Health and Sustainable Environment, College of Public Health, National Taiwan University, Taipei, Taiwan

${ }^{20}$ Department of Medicine, Baylor College of Medicine and Michael E. DeBakey Veterans Affairs Medical Center, Houston, Texas, USA

Acknowledgements The authors would like to express their special thanks to the staff of the Eighth Core Lab, Department of Medical Research, National Taiwan University Hospital, for the laboratory testing, and the staff in the Health Bureau of Lienchiang County, for their administrative support in the Matsu Islands. The study was also benefited from the inputs from Toshikazu Ushijima (Division of Epigenomics, National Cancer Center Research Institute, Tokyo, Japan) for the design of endoscopic surveillance using novel biomarkers.

Contributors $\mathrm{Y}-\mathrm{CL}$ and $\mathrm{H}-\mathrm{HC}$ had full access to the data and took responsibility for the integrity of the data, and the accuracy of the data analysis. Conception and design of the study: Y-CL and H-HC. Generation, collection, assembly, analysis and/ or interpretation of data: All authors. Pathological analysis: HC and C-TS. Statistical analysis: T-HC, W-JC, Y-CL and H-HC. Drafting or revision of the manuscript: T-HC, DYG, Y-CL and H-HC. Clinical reasoning and critical revision of the manuscript for important intellectual content: T-HC, DYG, Y-CL and H-HC. Administrative, technical or material support: All authors. Approval of the final version of the manuscript: Al authors. Study supervision: C-YL (policy development and administrative support), $\mathrm{Y}$-CL (programme execution and evaluation), and $\mathrm{H}-\mathrm{HC}$ (program execution and evaluation). Y-CL had the final responsibility for the decision to submit for publication.

Funding This study was supported by the Minister of Science and Technology, R.O.C. (105-2314-B-002-069-MY3 and 108-2314-B-002-140-MY3) and Taipei Institute of Pathology (TIP-106-005). The programme was also supported by grants from the Innovation and Policy Center for Population Health and Sustainable Environment (Population Health Research Center, PHRC), College of Public Health, National Taiwan University from the Ministry of Science and Technology (107-3017-
F-002-003), the Featured Areas Research Center Program within the framework of the Higher Education Sprout Project by the Ministry of Education (MOE) in Taiwan (NTU-107L9003), and the National Taiwan University Hospital (107-T11).

Disclaimer The funding sources had no role in study design, data collection, analysis or interpretation, report writing or the decision to submit this paper for publication

Competing interests DYG is a consultant for RedHill Biopharma and Phathom Pharmaceuticals regarding novel $\mathrm{H}$. pylori therapies and has received research support for culture of Helicobacter pylori. The rest authors have no conflicts of interest.

Patient and public involvement Patients and/or the public were involved in the design, or conduct, or reporting, or dissemination plans of this research. Refer to the Methods section for further details.

Patient consent for publication Not required.

Ethics approval All procedures followed were in accordance with the ethical standards of the responsible committee on human experimentation and with the Helsinki Declaration of 1964 and later versions. The Research Ethics Committee of National Taiwan University Hospital (IRB No: 940110) approved the protocol for this programme. Informed consent was obtained from all patients in the study.

Provenance and peer review Not commissioned; externally peer reviewed.

Data availability statement Data are available upon reasonable request. All data relevant to the study are included in the article or uploaded as online supplementary information.

Open access This is an open access article distributed in accordance with the Creative Commons Attribution Non Commercial (CC BY-NC 4.0) license, which permits others to distribute, remix, adapt, build upon this work non-commercially, and license their derivative works on different terms, provided the original work is properly cited, appropriate credit is given, any changes made indicated, and the use is non-commercial. See: http://creativecommons.org/licenses/by-nc/4.0/.

\section{ORCID iDs}

Amy Ming-Fang Yen http://orcid.org/0000-0002-0628-8768

Sherry Yueh-Hsia Chiu http://orcid.org/0000-0002-7207-7088

Han-Mo Chiu http://orcid.org/0000-0003-2786-8056

Ming-Shiang Wu http://orcid.org/0000-0001-5325-3974

Jaw-Town Lin http://orcid.org/0000-0001-9368-6141

David Y Graham http://orcid.org/0000-0002-6908-8317

Hsiu-Hsi Chen http://orcid.org/0000-0002-5799-6705

Yi-Chia Lee http://orcid.org/0000-0002-8160-1216

\section{REFERENCES}

1 Bray F, Ferlay J, Soerjomataram I, et al. GLOBOCAN estimates of incidence and mortality worldwide for 36 cancers in 185 countries. CA Cancer J Clin 2018;2018:394-424.

2 Tan MC, Balakrishnan M, Graham DY. Gastric cancer worldwide except Japan. In: Shiotani A, ed. Gastric cancer with special focus on studies from Japan. Singapore: Springer, 2018: 17-28.

3 Hunt RH, Camilleri M, Crowe SE, et al. The stomach in health and disease. Gut 2015:64:1650-68.

4 Graham DY. Helicobacter pylori update: gastric cancer, reliable therapy, and possible benefits. Gastroenterology 2015;148:719-31.

5 IARC Helicobacter pylori Working Group (2014). Helicobacter pylori Eradication as a Strategy for Gastric Cancer Prevention. Lyon, France: International Agency for Research on Cancer (IARC Working Group Reports, No. 8). Available: http:// publications.iarc.fr/Book-And-Report-Series/larc-Working-Group-Reports/-EmHelicobacter-Pylori-Em-Eradication-As-A-Strategy-For-Preventing-Gastric-Cancer2014. [Accessed 6 Aug 2019].

6 Fukase K, Kato M, Kikuchi S, et al. Effect of eradication of Helicobacter pylori on incidence of metachronous gastric carcinoma after endoscopic resection of early gastric cancer: an open-label, randomised controlled trial. Lancet 2008:372:392-7.

7 Choi IJ, Kook M-C, Kim Y-I, et al. Helicobacter pylori therapy for the prevention of metachronous gastric cancer. N Eng/ J Med 2018;378:1085-95.

8 Li W-Q, Zhang J-Y, Ma J-L, et al. Effects of Helicobacter pylori treatment and vitamin and garlic supplementation on gastric cancer incidence and mortality: follow-up of a randomized intervention trial. BMJ 2019;366:15016.

9 Choi IJ, Kim CG, Lee JY, et al. Family History of Gastric Cancer and Helicobacter pylori Treatment. N Engl J Med 2020:382:427-36.

10 Sugano K, Tack J, Kuipers EJ, et al. Kyoto global consensus report on Helicobacter pylori gastritis. Gut 2015;64:1353-67.

11 Malfertheiner P, Megraud F, O'Morain CA, et al. Management of Helicobacter pylori infection-the Maastricht V/Florence Consensus Report. Gut 2017:66:6-30.

12 Rabeneck L, Lansdorp-Vogelaar I. Assessment of a cancer screening program. Best Pract Res Clin Gastroenterol 2015:29:979-85. 
13 Lee Y-C, Lin J-T, Wu H-M, et al. Cost-Effectiveness analysis between primary and secondary preventive strategies for gastric cancer. Cancer Epidemiol Biomarkers Prev 2007;16:875-85.

14 Doorakkers E, Lagergren J, Santoni G, et al. Helicobacter pylori eradication treatment and the risk of Barrett's esophagus and esophageal adenocarcinoma. Helicobacter 2020;25:e12688.

15 Hu K-C, Wu M-S, Chu C-H, et al. Decreased colorectal adenoma risk after Helicobacter pylori eradication: A retrospective cohort study. Clin Infect Dis 2019;68:2105-13.

16 Megraud F, Coenen S, Versporten A, et al. Helicobacter pylori resistance to antibiotics in Europe and its relationship to antibiotic consumption. Gut 2013;62:34-42.

17 Sung JJY. Population-based screening and mass eradication of Helicobacter pylori infection to prevent gastric cancer: There are more questions than answers. J Gastroenterol Hepatol 2020;35:521-2.

18 Lee Y-C, Wu H-M, Chen TH-H, et al. A community-based study of Helicobacter pylori therapy using the strategy of test, treat, retest, and re-treat initial treatment failures. Helicobacter 2006;11:418-24.

19 Lee $\mathrm{Y}-\mathrm{C}$, Chen $\mathrm{TH}-\mathrm{H}$, Chiu H-M, et al. The benefit of mass eradication of Helicobacter pylori infection: a community-based study of gastric cancer prevention. Gut 2013:62:676-82.

20 Chiang T-H, Chiu SY-H, Chen SL-S, et al. Serum pepsinogen as a predictor for gastric cancer death: a 16-year community-based cohort study. J Clin Gastroenterol 2019:53:e186-93.

21 Liou J-M, Chen C-C, Chang C-Y, et al. Sequential therapy for 10 days versus triple therapy for 14 days in the eradication of Helicobacter pylori in the community and hospital populations: a randomised trial. Gut 2016;65:1784-92

22 Cook TD, Campbell DT. Quasi-Experiments Interrupted Time-Series Designs. In: QuasiExperimentation: Design \& Analysis Issues for Field Settings. Boston: Houghton Mifflin Co, 1979: 207-32.

23 Dixon MF, Genta RM, Yardley JH, et al. Classification and grading of gastritis. The updated Sydney system. International workshop on the histopathology of gastritis, Houston 1994. Am J Surg Pathol 1996;20:1161-81.

24 Rugge $M$, Meggio A, Pennelli $G$, et al. Gastritis staging in clinical practice: the OLGA staging system. Gut 2007;56:631-6.

25 Capelle LG, de Vries AC, Haringsma J, et al. The staging of gastritis with the OLGA system by using intestinal metaplasia as an accurate alternative for atrophic gastritis. Gastrointest Endosc 2010;71:1150-8.

26 Chiang CJ, Chen YC, Chen CJ, et al. Taiwan cancer registry Task force. cancer trends in Taiwan. Jpn J Clin Oncol 2010;40:897-904.

27 National Cancer Registry. On-Line interactive data acquisition system, health promotion administration, Ministry of health and welfare, Taiwan. Available: https:// cris.hpa.gov.tw/ [Accessed 20 Feb 2020].

28 Cancer Registry Annual Report. Health promotion administration, Ministry of health welfare, Taiwan, 2017. Available: https://www.hpa.gov.tw/Pages/Detail.aspx?nodeid= 269\&pid=12235 [Accessed 6 Jul 2020].

29 Department of Household Registration, Ministry of the Interior, Taiwan. Household registration statistics data analysis. Available: https://www.ris.gov.tw/app/portal/346 [Accessed 20 Feb 2020].
30 Bosman FT, Carneiro F, Hruban RH. WHO classification of tumours of the digestive system. Lyon: IARC, 2010

31 Congdon PD. Bayesian hierarchical models: with applications using R. Second edn. Chapman and Hall/CRC Press, 2019.

32 Arnold M, Park JY, Camargo MC, et al. Is gastric cancer becoming a rare disease? a global assessment of predicted incidence trends to 2035. Gut 2020;69:823-9.

33 Jun JK, Choi KS, Lee H-Y, et al. Effectiveness of the Korean National cancer screening program in reducing gastric cancer mortality. Gastroenterology 2017:152:1319-28.

34 Lee Y-C, Chiang T-H, Chou C-K, et al. Association between Helicobacter pylori eradication and gastric cancer incidence: a systematic review and meta-analysis. Gastroenterology 2016;150:1113-24.

35 Ford AC, Yuan Y, Moayyedi P. Helicobacter pylori eradication therapy to prevent gastric cancer: systematic review and meta-analysis. Gut 2020;69:2113-21.

36 Chen $\mathrm{Q}$, Liang $\mathrm{X}$, Long $\mathrm{X}$, et al. Cost-effectiveness analysis of screen-and-treat strategy in asymptomatic Chinese for preventing Helicobacter pylori-associated diseases. Helicobacter 2019;24:e12563.

37 Wong BC-Y, Lam SK, Wong WM, et al. Helicobacter pylori eradication to prevent gastric cancer in a high-risk region of China: a randomized controlled trial. JAMA 2004;291:187-94.

38 Leung WK, Lin S-R, Ching JYL, et al. Factors predicting progression of gastric intestina metaplasia: results of a randomised trial on Helicobacter pylori eradication. Gut 2004;53:1244-9.

39 Liou J-M, Malfertheiner $\mathrm{P}$, Lee $\mathrm{Y}-\mathrm{C}$, et al. Screening and eradication of Helicobacter pylori for gastric cancer prevention: the Taipei global consensus. Gut 2020:69:2093-112.

40 Blaser MJ. Antibiotic use and its consequences for the normal microbiome. Science 2016:352:544-5

41 Bair M-J, Chuang S-L, Lei W-Y, et al. Planning mass eradication of Helicobacter pylori infection for indigenous Taiwanese peoples to reduce gastric cancer. J Gastroenterol Hepatol 2020;35:609-16

42 Graham DY, Lee Y-C, Wu M-S. Rational Helicobacter pylori therapy: evidencebased medicine rather than medicine-based evidence. Clin Gastroenterol Hepatol 2014; 12:177-86

43 Liou JM, Chen CC, Chang CM, et al. Taiwan Gastrointestinal Disease and Helicobacter Consortium. Long-term changes of gut microbiota, antibiotic resistance, and metabolic parameters after Helicobacter pylori eradication: a multicentre, open-label, randomised trial. Lancet Infect Dis 2019;19:1109-20.

44 Guo Y, Zhang Y, Gerhard M, et al. Effect of Helicobacter pylori on gastrointestinal microbiota: a population-based study in Linqu, a high-risk area of gastric cancer. Gut 2020;69:1598-607.

45 Rugge M, Meggio A, Pravadelli C, et al. Gastritis staging in the endoscopic follow-up for the secondary prevention of gastric cancer: a 5-year prospective study of 1755 patients. Gut 2019:68:11-17.

46 Chiang Tsung-Hsien, Maeda M, Yamada H, et al. Risk Stratification for Gastric Cancer after Helicobacter pylori Eradication: A Population-based Study on Matsu Islands. J Gastroenterol Hepatol 2020 